\title{
Excimer Laser Phototherapeutic Keratectomy for Corneal Dystrophies
}

\author{
a report by \\ Christopher J Rapuano, MD \\ Co-director, Cornea Service and Refractive Surgery Department, Wills Eye Institute and Professor of \\ Ophthalmology, Jefferson Medical College, Thomas Jefferson University \\ DOI: $10.17925 /$ USOR.2006.00.00.30
}

Christopher J Rapuano, MD, joined the Cornea Service at Wills Eye Hospital, Philadelphia, US, after his fellowship in 1991. He is a nationally and internationally recognized expert in corneal diseases, with a special interest in refractive surgery. He feels that cornea specialists are particularly skilled at performing laser-assisted in situ keratomileusis (LASIK), which essentially involves reshaping the cornea. Dr Rapuano performs al pre- and post-operative care for his LASIK patients and when complications occasionally occur other LASIK doctors refer their patients to him for his expertise and care. He has published several books and over 100 research papers in the medical literature on corneal diseases and excimer laser phototherapeutic keratectomy (PTK) surgery. During his ophthalmology residency, he co-authored a bestselling textbook in ophthalmology, The Wills Eye Manual. Dr Rapuano serves on many committees of the American Academy of Ophthalmology (AAO) and on the editorial boards of numerous peer-reviewed ophthalmology journals, including the American Journal of Ophthalmology. His thesis was recently accepted to become a member of the American Ophthalmalogical Society.
While there are seemingly weekly advances in the genetic classification of corneal dystrophies, clinicians generally differentiate them by their anatomic depth. From a practical standpoint, the level of the pathology in the cornea directly impacts both the patient's symptoms and their treatment options. Anterior disease is more likely to cause painful erosions and corneal epithelial irregularity. Mid-corneal disease tends to cause decreased vision more than pain. Posterior corneal disease tends not to cause symptoms until corneal edema develops. The more anterior the pathology, the more amenable it is to superficial keratectomy with a blade, or more recently, with the excimer laser. ${ }^{1}$

Anterior corneal dystrophies, such as anterior basement membrane dystrophy (ABMD) and the corneal dystrophies of Bowman's membrane (classically Reis-Bücklers' dystrophy), often cause disruption of the most superficial layer of the cornea, which can result in recurrent erosions. Recurrent erosions are painful breaks in the corneal epithelium, which can last from minutes to days, depending on their size. One of the worst aspects of recurrent erosions is that they are unpredictable. They may not occur for weeks to months, but then develop on the morning of an important event such as a business meeting or family wedding. Not only can erosions incapacitate patients due to pain and decreased vision, but they also greatly increase the risk of corneal infection. Depending on the degree of corneal irregularity and opacity, anterior dystrophies can also cause decreased vision even without recurrent erosion.

Corneal dystrophies involving the stroma - the 'body' of the cornea-such as granular, lattice, macular, and Schnyder's crystalline dystrophy, tend to cause fewer painful erosions, but can certainly affect the vision depending on the degree of opacity. Multiple separate stromal opacities cause fewer disturbances to the vision than confluent opacities. Posterior corneal dystrophies, for example endothelial dystrophy, Fuchs' dystrophy and posterior polymorphous dystrophy, affect the endothelium - the back layer of the cornea.
The endothelial cells pump fluid out of the cornea to keep it clear. When the endothelial cells are severely damaged, corneal edema develops, at which point the vision is affected and eventually the eye can become painful.

The argon-fluoride excimer laser uses $193 \mathrm{~nm}$ wavelength ultraviolet light to remove corneal tissue. It can remove approximately $1 / 2,000$ th of the corneal thickness per laser pulse and the spot size can be adjusted from less than $1 \mathrm{~mm}$ to almost the entire corneal diameter. The number of pulses delivered determines the depth of the ablation. The excimer laser removes tissue by breaking molecular bonds with minimal damage to adjacent or deep corneal tissue. It is US Food and Drug Administration (FDA)-approved to treat nearsightedness, farsightedness, and astigmatism by reshaping the corneal surface through tissue removal. Tissue can be removed from the superficial corneal stroma (surface ablation) or the mid-stroma (laser in situ keratomileusis-LASIK) after a partial thickness corneal flap is created and moved to the side. The excimer laser is also FDA-approved to remove anterior corneal pathology and smooth the corneal surface to improve visual function and painful symptoms in a procedure termed 'phototherapeutic keratectomy' (PTK). Excimer laser PTK is not useful for posterior corneal dystrophies. While the number of PTK procedures is tiny compared with the number of surface ablation and LASIK procedures, PTK can significantly improve the vision, pain and discomfort and consequently quality of life for many patients with superficial and anterior stromal dystrophies. ${ }^{2}$

PTK for corneal dystrophies is as much an art as a science. Not only are different dystrophies treated differently, but since each dystrophy has a wide spectrum of corneal involvement, different corneas with the same dystrophy are also treated differently.

In patients with ABMD, where the corneal epithelium is loose or irregular, causing decreased vision, painful erosions, or both, all the abnormal 
epithelium is removed manually with a cellulose sponge or a blade. The excimer laser is then used to remove about $1 \%$ of the superficial stroma (5-6 microns, partway through Bowman's membrane), which allows for stronger and smoother adhesion of the new epithelial layer when it grows back in. Postoperative management includes topical antibiotics, cycloplegics and close follow-up until the epithelial defect has healed. Effective pain management is critical as these large epithelial defects can be excruciating and may take several days to heal. PTK is reportedly $80-95 \%$ successful when used to treat both painful recurrent erosions and decreased vision associated with ABMD. ${ }^{3-6}$

Corneal dystrophies of Bowman's membrane, most commonly Reis-Bücklers' dystrophy, involve the superficial stroma and cause poor vision from corneal irregularity and opacification. However, their most devastating symptom is painful recurrent erosions, which can begin in early childhood; even in infancy. When the epithelium is irregular, it is removed in a similar manner to ABMD and the laser treatment applied to the superficial Bowman's membrane and anterior stroma. When it is regular, the excimer laser treatment is performed through the epithelium and through Bowman's membrane and into the anterior stroma. In this case the epithelium is acting as masking agent, theoretically leading to a smoother stroma. How much of the anterior stroma to remove in any given eye is the big question. The answer, which is easier to say than to do, is to just remove the bulk of the opacity to clear the majority of the central cornea. The caveats are:

- ablate the least amount of tissue to clear the majority of the central cornea; and

- the cornea does not need to be crystal clear after the procedure to be successful.

Deep ablations tend to induce more post-operative haze/scar and cause greater corneal flattening, which induces hyperopia. Since the optics of the laser system operating microscope are not ideal, sitting the patient up a few times during the laser treatment to examine them at the slit lamp will determine whether additional treatment is needed.

It is remarkable how much better the vision can become after PTK for Reis-Bücklers' dystrophy, although it often requires a change in glasses prescription. Invariably, what patients appreciate the most is the reduction (or elimination, ideally) of the painful recurrent erosions. While PTK is routinely successful in patients with Reis-Bücklers' dystrophy,
Figure I: Recurrent Reis-Bücklers Dystrophy Five Years After Successful Excimer Laser PTK

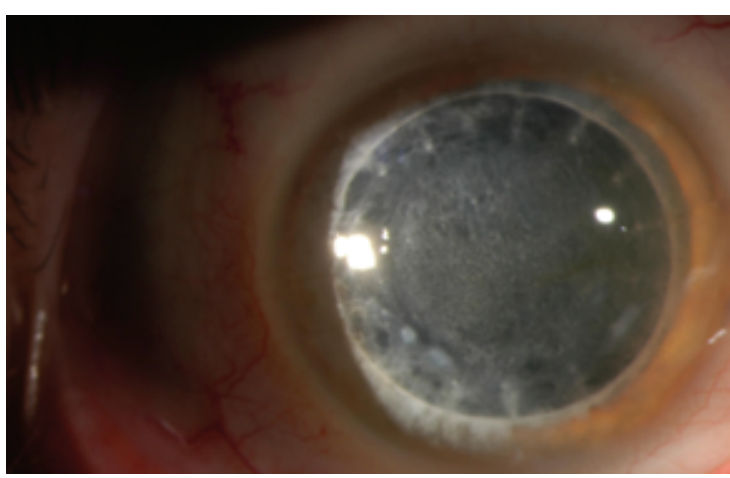

Figure 2: Two Weeks after Repeat PTK with Adjunctive Mitomycin C for Recurrent Reis-Bücklers Dystrophy, with Significant-Although not Perfect-Clearing of the Visual Axis and Considerable Improvement in Vision

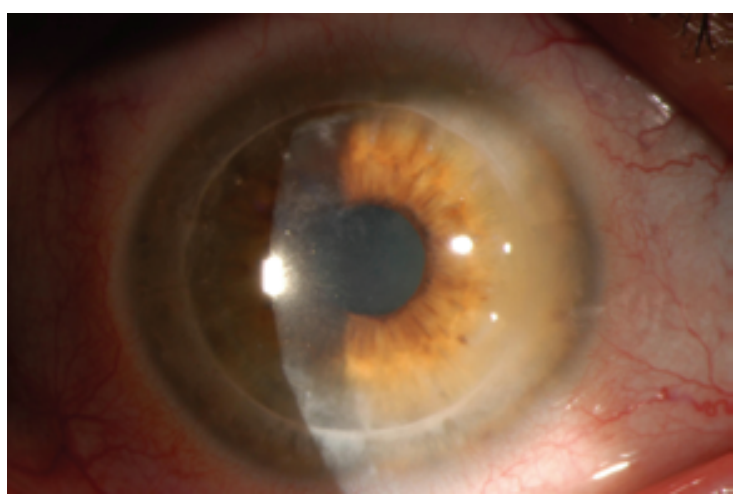

Figure 3: Granular Dystrophy with Moderate Breadcrumb-like Opacities in the Central Cornea

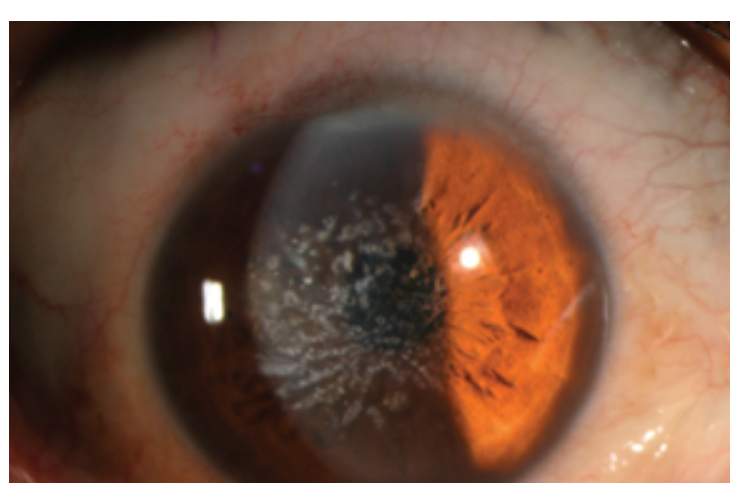

the dystrophy (and its symptoms) almost always recur, and often quite quickly, within one to five years. Fortunately, PTK can typically be repeated, although the dystrophy recurs after the repeat treatments too (See Figures 1 and 2).

There is some evidence that a short application of topical mitomycin $\mathrm{C}$, a potent anti-scarring agent, 
Figure 4: Five Months after PTK for Granular Dystrophy with Substantial Improvement in Corneal Opacity, Visual Acuity and Symptoms

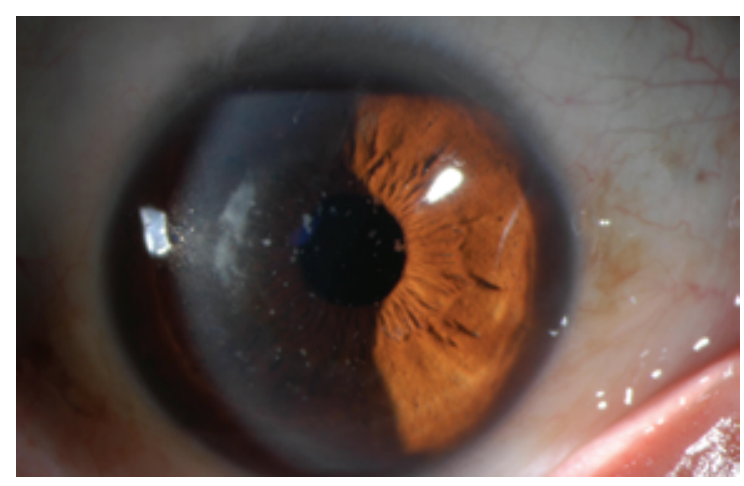

Note: some residual deep granules centrally and the post-ptk fibrosis at the edge of the ablation from 9-10.30 o'clock, neither of which greatly affect the vision.

may be useful in prevention or slowing of recurrence of Reis-Bücklers' dystrophy and other corneal pathologies after PTK. ${ }^{8-10}$

PTK is also useful for deeper stromal dystrophies. ${ }^{11-13}$ Here, too, the exact amount of tissue to remove is difficult to determine pre-operatively. The author performed a study to evaluate whether ultrasound biomicroscopy (UBM) would be helpful in estimating how much tissue to remove, but unfortunately, it was not. ${ }^{14}$ Hopefully, newer corneal imaging techniques, such as even higher frequency ultrasound, Scheimpflug photography and ocular coherence tomography, will be more helpful to surgeons.

Currently, the best technique is to perform a careful pre-operative slit lamp examination and estimate the depth of the bulk of the opacity, for example 10 or $15 \%$ into the cornea. Ultrasound pachymetry is then performed to measure the entire corneal thickness.
The depth of the pathology can then be estimated, e.g. $15 \%$ of a 500 -micron cornea is 75 microns. At the laser, the optometrist can ablate about two-thirds of the depth estimated pre-operatively, in this case, 50 microns. At this point, the patient is examined sitting up at a slit lamp to see the effect of the ablation on the corneal opacity. Occasionally, the majority of the opacity is gone, and the treatment is stopped there. More often than not, significant opacity remains and additional laser treatment is continued. The patient may be examined at the slit lamp several times during a single procedure, to provide the best chance of removing enough of the opacity to improve the visual symptoms, with the least chance of inducing significant corneal haze/scar and flattening (see Figures 3 and 4).

The post-operative treatment is similar to after PTK for ABMD. Stromal dystrophies can also recur after PTK, but they tend to recur much more slowly than Reis-Bücklers' dystrophy. Fortunately, repeat PTK is often successful for a least a while. ${ }^{7}$

The use of PTK to treat corneal dystrophies has greatly benefited patients over the past 15 years. Many fewer corneal transplants are currently being performed for dystrophy patients since the advent of excimer laser PTK. Additionally, patients with milder forms of the dystrophies - that are not severe enough for a corneal transplant but are disturbing the visual or comfort-are undergoing treatment with PTK with significant improvement in their symptoms. PTK has been a wonderful addition to the corneal surgeon's armamentarium for the treatment of anterior and stromal corneal dystrophies. With improvements in diagnostic and laser treatment technology, PTK results will only get better in the future.

References

1. Rapuano CJ, Curr Opin Ophthalmol (2001); 12:pp. 288-293.

2. Rapuano CJ, Cornea (1997); 16:pp. 151-157.

3. Sridar MS, Rapuano CJ, Cosar CB, et al., Ophthalmology (2002); 109:pp. 674-670.

4. Rashad KM, Hussein HA, El-Samadouny MA, et al., J Refract Surg (2001); 17:pp. 511-518.

5. Cavanaugh TB, Lind DM, Cutarelli PE, et al., Ophthalmology (1999); 106:pp. 971-976.

6. Tzelikis PF, Rapuano CJ, Hammersmith KM, et al., Am J Ophthalmol (2005); 140:pp. 308-310.

7. Dinh R, Rapuano CJ, Cohen EJ, Laibson PR, Ophthalmology (1999); 106:pp. 1,490-1, 497.

8. Ayres BD, Hammersmith KM, Laibson PR, Rapuano CJ, Am J Ophthalmol (2006); 142:pp. 490-492.

9. Miller A, Solomon R, Bloom A, et al., Cornea (2004); 23:pp. 732-735.

10. Marcon AS, Rapuano CJ, Cornea (2002); 21:pp. 828-830.

11. Das S, Langenbucher A, Seitz B, J Refract Surg (2005); 21:pp. 727-731.

12. Hafner A, Langenbucher A, Seitz B, Am J Ophthalmol (2005); 140:pp. 392-396.

13. Paparo LG, Rapuano CJ, Raber IM, et al., Cornea (2000); 19:pp. 343-347.

14. Rapuano CJ, Trans Am Ophthalmol Soc (2003); 101:pp. 371-399. 